\section{Civilizar \\ humanas}

ISSN 1657-8953 e-ISSN: 2619-189X

\title{
La flexibilización del derecho moral de integridad por la jurisprudencia andina*
}

\author{
Moral Rights' Flexibilization by Andean Case Law
}

Marcela Palacio Puertas

Edwin Jesith Bernal Ramírez ${ }^{\ddagger}$

* Artículo de investigación realizado en el grupo Investigaciones jurídicas y Socio-Jurídicas, en la línea de investigación: Derecho Privado y Actualidad de las Relaciones entre Particulares, financiado por la Universidad Santo Tomás, Seccional Tunja.

$\S$ Doctora en Derecho de la American University Washington College of Law. Investigadora de la Universidad Santo Tomás, Seccional Tunja.

Correo electrónico:

marcela.palacio@usantoto.edu.co.

(iD) 0000-0002-6338-0093

‡ Abogado de la Universidad Externado de Colombia, Doctorante en Estudios Sociales de la Universidad Distrital Francisco José de Caldas. Docente tiempo completo de la Facultad de Derecho de la Universidad Militar Nueva Granada.

Correo electrónico:

edwin.bernal@unimilitar.edu.co

(iD) 0000-0002-2876-7989

Cómo citar:

Palacio Puerta, M., y Bernal Ramírez, E. (2020). La Flexibilización del Derecho Moral de Integridad por la Jurisprudencia Andina. Civilizar: Ciencias Sociales y Humanas, 20(38), 81-94. https://doi. org/10.22518/jour.ccsh/2020.1a01

Recibido: 12/09/2019

\section{Resumen}

El derecho moral de autor en el ordenamiento jurídico colombiano se ha entendido como un derecho fundamental que busca proteger esa conexión especial existente entre el autor y su obra. No obstante, debido a que el derecho moral se encuentra también regulado por la Decisión Andina 351 de la Comisión de la Comunidad Andina, es la jurisprudencia del Tribunal Andino la más pertinente para determinar el verdadero alcance de su protección. De esta manera, el presente artículo busca evidenciar que la interpretación prejudicial 47-IP-17 flexibilizó la protección del derecho moral de integridad para cierto tipo de obras, lo cual produjo un impacto en el ámbito doméstico: la necesidad de revocar la decisión de primera instancia en el caso Gabriel Antonio Calle.

\section{Palabras clave}

Derecho moral de autor, derecho de integridad, jurisprudencia andina, flexibilización

\section{Abstract}

Author's moral rights under the Colombian legal system are protected as a fundamental right that seeks to protect the special connection between the author and their work. However, since moral rights are regulated under the Andean Law, it is the Andean Court of Justice's jurisprudence the one to determine their scope of protection. In this way, this paper aims to argue that decision 47-IP-17 relaxed the threshold of protection of the moral right of integrity for certain types of works, having as an impact at the domestic sphere the need to revoke the decision of first instance in the Gabriel Antonio Calle case.

\section{Keywords}

Moral rights, integrity right, Andean jurisprudence, flexibilization 


\section{Introducción}

En el ordenamiento jurídico colombiano, los derechos morales de autor protegen la especial conexión entre el autor y su obra. El fundamento de esta regulación se encuentra en que, al devenir la calidad de autor de su condición de ser humano, el autor imprime su alma / personalidad en su creación. Por esta misma razón, la Corte Constitucional ha catalogado estos derechos como derechos fundamentales. Dicha posición parece ser concordante con la normativa regional, pues la Decisión Andina 351 de 1993 ha protegido los derechos morales de una manera que sobrepasa los mínimos de protección establecidos por el Convenio de Berna de 1971.

La Decisión Andina 351 de 1993 establece en el artículo 11, sobre la protección al derecho moral, que: "el autor tiene el derecho inalienable, inembargable, imprescriptible e irrenunciable [sobre su obra]”. Dicho tenor concuerda con la legislación nacional -Ley 23 de 1982-, la cual establece en su artículo 30 que, "el autor tendrá sobre su obra un derecho perpetuo, inalienable, e irrenunciable". De lo establecido en textos normativos, es claro que, para la norma andina - bajo un criterio gramatical de interpretación-, el derecho moral es inherente al autor, al no ser posible su renuncia o enajenación; del mismo modo que lo ha entendido la Dirección Nacional de Derecho de Autor, tanto en conceptos ${ }^{1}$ como en sus funciones jurisdiccionales, tal como se explicará más adelante.

La Decisión Andina 351 es parte del ordenamiento jurídico de la Comunidad Andina (CAN), la cual -desde la modificación establecida por el Protocolo de Trujillo de 1996- aparece como una integración en busca del desarrollo social y económico de manera armoniosa y equilibrada para sus integrantes. Como parte del ordenamiento jurídico andino, la Decisión es de aplicación prevalente sobre la norma nacional directa e inmediata. Adicionalmente, el único ente autorizado para interpretar la norma andina es el Tribunal de Justicia de la Comunidad Andina (TJCA) a través de interpretaciones prejudiciales $^{2}$. Finalmente, el juez nacional está obligado a acoger dicha interpretación.

Tal como lo establece la Decisión 500 de 2017, el Tribunal Andino de Justicia propende por criterios hermenéuticos funcionales, tales como el sistemático, el teleológico y de la ratio legis, al momento de resolver las interpretaciones de los Estados miembros. Bajo estos criterios de interpretación, en el 2017, el Tribunal de Justicia Andino expidió una interpretación prejudicial sobre el artículo 11 que no parece concordante con la literalidad que expresa la Decisión, como se explicará más adelante. Esta situación lleva a preguntarse ¿cómo ha impactado la jurisprudencia del Tribunal Andino de Justicia el alcance del derecho moral de integridad? En este sentido, el objetivo del presente consiste en identificar el estado actual del alcance del derecho moral de integridad luego de ser dictada la interpretación prejudicial 47-IP-17.

El interrogante anterior cobra vital importancia en vista de que, la mayoría de las veces, el alcance o la forma como las interpretaciones prejudiciales pueden impactar las decisiones de los jueces nacionales y el entendimiento de la norma andina son desconocidas para litigantes y doctrinantes colombianos debido a la falta de publicación de las sentencias judiciales domésticas que no provengan de los altos tribunales ${ }^{3}$. Si bien es cierto que las interpretaciones prejudiciales son publicadas por el Tribunal Andino de Justicia, estas no resuelven los casos en concreto, ni pueden interpretar normas nacionales; por lo tanto, el verdadero impacto de las interpretaciones solo se puede observar en la sentencia nacional. Ante este escenario, el presente artículo constituye un primer avance para evidenciar el impacto de la hermenéutica andina aplicada en la interpretaciones prejudiciales en el alcance del derecho de autor, y más específicamente en el derecho moral colombiano.

\section{Metodología}

La presente investigación es de corte jurídico dogmático y hermenéutico dado que analiza la interpretación de la norma andina en relación con los derechos morales de autor. Para el desarrollo de este artículo de investigación, se utilizó el método de interpretación sistemático; se consideraron los diferentes componentes normativos regionales que regulan los derechos de autor y el sistema andino de justicia: la Decisión Andina 351, el Tratado de Constitución del Tribunal Andino de Justicia y la Decisión 500 de 2017, así como los casos jurisprudenciales de Gabriel Antonio Calle vs. Centro Comercial San Diego y la interpretación prejudicial 47-IP- 17.

Para la ubicación de las fuentes, se utilizó la técnica cualitativa de recopilación de información denominada investigación documental. A través del uso de técnicas bibliográficas y hemerográficas, se investigó en libros, bases de datos especializadas, 
prensa, revistas de investigación, jurisprudencia y regulación en derecho comparado. Finalmente, para el análisis de las fuentes identificadas, se utilizaron técnicas cualitativas, como la lectura y el análisis documental, en busca de encontrar la relación y el impacto de la jurisprudencia andina en el alcance del derecho moral de autor.

\section{Los derechos morales de autor y su regulación a nivel internacional}

El derecho de autor es una institución jurídica que le confiere al autor un conjunto de prerrogativas patrimoniales y morales por el hecho de crear una obra artística literaria o científica. Sin ser relevante el mérito o la finalidad, se requiere que sea original y sea susceptible de ser difundida o reproducida. Al respecto, los derechos patrimoniales denotan las facultades que tiene el titular originario o derivado para explotar económicamente la obra. Estos derechos pueden permitir, o prohibir la reproducción, comunicación pública, la distribución, la traducción o adaptación de la obra (artículo 13, Decisión 351 de 1993). El derecho de autor, mencionó Le Chapelier, es la más sagrada, legítima, irrefutable, y si se permite decirlo, la más personal de todas las propiedades; es la obra fruto del pensamiento de un escritor; sin embargo, es una propiedad de un género completamente diferente de las demás propiedades (1791, p. 16).

Por otra parte, los derechos morales de autor son entendidos como el vínculo estrecho entre el autor y su obra, y permiten al autor ejercer un conjunto de prerrogativas extrapatrimoniales como lo son el derecho a reivindicar la paternidad de su obra en todo tiempo, oponerse a cualquier modificación de la obra que afecte el decoro de esta o la reputación del autor, mantener la obra en su esfera privada o darla a conocer al público, y solicitar el retiro de la obra del mercado, incluso cuando esta ha sido publicada (Villaba, 2019). Estos derechos buscan proteger la creación intelectual y la esencia de la personalidad del autor manifestada en su obra.

Los derechos morales de autor son de carácter personalísimo; su "finalidad última es la tutela de la dignidad intelectual de los autores" (Parra, 2014, p. 56). La noción de derechos morales ha sido una construcción desde la filosofía, la doctrina y la jurisprudencia al vincularlos con la teoría de la personalidad (Hegel, 2012), "según la cual el individuo manifiesta su voluntad creativa y se apropia de esas obras que imprimen su personalidad al exteriorizar su identidad" (Bernal, 2019). En efecto, según esta teoría, el derecho moral emana de la personalidad del autor: de crear y hacer respetar esta personalidad manifestada en la obra artística o literaria. Respecto a sus características; ; el derecho moral de autor se transmite mortis causa a los herederos del autor originario, al menos en el caso del derecho de paternidad y de integridad. Este es un derecho desprovisto de valoración económica; por lo cual, se encuentra por fuera del comercio. Es decir, es un derecho inalienable e inembargable. Además de ser un derecho oponible erga omnes (Binctin, 2013), e inalienable e indisociable de la calidad del autor (Almansi et al., 2010).

En el ámbito jurisprudencial, Francia fue el primer país en el cual los operadores jurídicos otorgaron protección a los intereses espirituales del autor. En un fallo del 17 de agosto de 1814, del Tribunal de Seine reconoció la integridad de la obra en el derecho (Strômholm, 1967, como se cita en Goudreau, 1994, p. 405). De igual manera, se resaltan los primeros proyectos de ley para salvaguardar el derecho moral de autor. Plaisant ${ }^{5}$ fue uno de los pioneros redactores de la proposición para proteger esta categoría de derechos. Este autor reconocía que: si el autor es libre de renunciar -según los contratos-al beneficio de su obra, no le está permitido romper el vínculo espiritual establecido entre la persona y las expresiones literarias o artísticas que permanecen como su espejo permanente. El interés que se debe salvaguardar a través de las vicisitudes de la publicación, la exposición, el espectáculo de su obra es la de su propia personalidad. Este un derecho que domina al comercio: tiene el carácter moral (Plaisant, 1921, mayo 15, como se cita en Raizon, 2014, p. 21).

\section{Reconocimiento internacional de los derechos morales de autor}

En el contexto internacional, los derechos morales obtienen reconocimiento por primera vez en el Convenio de Berna para la Protección de las Obras Literarias y Artísticas. Este pacto se convirtió en un importante instrumento internacional en materia de protección a los derechos de autor, el cual, a su vez, constituye un esfuerzo de armonización internacional en esta especialidad del derecho.

Respecto a la génesis de la Convención de Berna, se tuvo en cuenta como marco principal la Asociación Literaria y Artística Internacional, fundada en 1878 bajo la presidencia honoraria del escri- 
tor Víctor Hugo. En un congreso celebrado en Roma en 1882, se expresó el deseo de que una conferencia diplomática se reuniera para crear los fundamentos de una unión de propiedad literaria: se trataría de establecer entre los Estados signatarios una legislación uniforme de derecho de autor (Desbois, 1960). Aunque no fue fácil su consecución, en 1886 el Convenio de Berna se erigió como el primer tratado multilateral en la materia, que estableció los mínimos estándares de protección que todos los países signatarios debían otorgar.

Adicionalmente, cada Estado contratante se comprometía a proporcionar la protección otorgada por su propio ordenamiento jurídico, también, a las obras del intelecto elaboradas por ciudadanos de otros países miembros de la convención. La protección fue declarada automática, toda vez que está prohibida cualquier formalidad constitutiva del derecho de autor capaz de obstaculizar el disfrute y el ejercicio de los derechos de autor, como, por ejemplo, la introducción de normas de protección para autores extranjeros subordinadas al registro de la obra (Zucconelli, 2014, p. 52).

Respecto a los derechos morales, cabe resaltar que el texto inicial de la Convención de Berna de 1886 no reconocía los derechos morales de autor; fue hasta la Conferencia de Roma de 1928 cuando se introdujo el artículo 6 Bis, que consagra los derechos morales de autor. Dicho artículo reconoció a los autores de los países miembros de la Unión dos derechos: 1) reivindicar la paternidad de la obra y 2) oponerse a cualquier mutilación o modificación de la obra que cause perjuicio al honor o a la reputación, conocido este último como derecho de integridad. Dichos derechos se crearon de naturaleza independiente a los derechos patrimoniales de autor. Es decir, aún después de haber cedido sus derechos patrimoniales, el autor conservaría estas prerrogativas. De igual manera, el Convenio de Berna determinó que esos dos derechos debían permanecer luego de la muerte del autor; por lo menos mientras se mantuviesen vigentes los derechos patrimoniales. El Convenio dictó establecer medios judiciales para proteger estos derechos por la legislación interna del país donde se reclame su protección.

De igual manera, la protección a los derechos morales de autor es reiterada en otros dos tratados internacionales: 1) La Declaración Universal de los Derechos Humanos de 1948 y 2) el Pacto de Derechos Económicos, Sociales y Culturales de 1966. En la Declaración Universal de los Derechos Humanos -DUDH- del 10 de diciembre de 1948, artículo 27, se consagra el derecho de los individuos para participar en la vida cultural y en el progreso científico. En el inciso segundo, se establece que: "toda persona tiene derecho a la protección de los intereses morales y materiales que le correspondan por razón de las producciones científicas, literarias o artísticas de que sea autora" (DUDH, artículo 27 diciembre de 1948).

La versión definitiva de la Declaración Universal de los Derechos Humanos fue elaborada por el delegado de Francia, el jurista René Cassin, cuya presencia en "el comité fue determinante para la posterior inclusión de los derechos de autores e inventores en el texto definitivo del artículo 27. Sin embargo, esta cláusula no fue incorporada sino hasta el tramo final del trabajo de redacción de la Declaración" (Busaniche, 2019, p. 5). Cabe destacar que la inclusión del inciso segundo del artículo 27 de la DUDH desató arduos debates. Inicialmente, este apartado fue rechazado por ser considerado inapropiado para ser parte de un documento de derechos humanos. En efecto, se reitera el protagonismo de René Cassin para incluir en el texto de la declaración una protección a los derechos morales de autor y el reconocimiento a una justa remuneración por su trabajo (Shaver, 2010).

Posteriormente, en el Pacto Internacional de Derechos Económicos, Sociales y Culturales (PIDESC) de 1966, en el artículo 15, inciso c, se reitera el derecho "de toda persona a beneficiarse de la protección de los intereses morales y materiales que le correspondan por razón de las producciones científicas, literarias o artísticas de que sea autora”. Ahora bien, respecto a la interpretación del artículo 15, existen diferentes observaciones del Comité de PIDESC que señalan la protección a los derechos morales como derecho humano. Esta protección es solo para las personas autoras y no para personas jurídicas ni causahabientes de los autores. Los derechos reconocidos en el artículo 15 del Pacto no deben impedir el ejercicio de otros derechos reconocidos en este instrumento de derechos humanos. En particular, la Observación general N. ${ }^{\circ} 17$ de 2005 señala:

Es importante pues no equiparar los derechos de propiedad intelectual con el derecho humano reconocido en el apartado c) del párrafo 1 del artículo 15. El derecho humano a beneficiarse de la protección de los intereses morales y materiales del autor se reconoce en los instrumentos internacionales per- 
tinentes comprenden, entre otros, el Convenio de París para la Protección de la Propiedad Industrial, en su última versión revisada en 1967; el Convenio de Berna para la Protección de las Obras Literarias y Artísticas, en su última versión revisada en 1971; la Convención Internacional sobre la protección de los artistas intérpretes o ejecutantes, los productores de fonogramas y los organismos de radiodifusión (la "Convención de Roma"); el Tratado de la OMPI sobre Derecho de Autor; el Tratado de la OMPI sobre Interpretaciones o Ejecuciones y Fonogramas (que entre otras cosas prevé la protección internacional de los artistas intérpretes o ejecutantes de "expresiones del folclore"), el Convenio sobre la Diversidad Biológica; la Convención Universal sobre Derechos de Autor, en su última versión revisada en 1971; y el Acuerdo sobre los Aspectos de los Derechos de Propiedad Intelectual Relacionados con el Comercio (ADPIC) de la OMC.

\section{(...)}

El Comité considera que sólo el "autor", lo que significa el creador -ya sea hombre o mujer, individuo o grupo- de producciones científicas, literarias o artísticas como, por ejemplo, escritores, artistas e inventores, entre otros, se puede beneficiar de la protección que ofrece el Humanos; y el artículo 9 de la Carta Africana de Derechos Humanos y de los Pueblos (Carta de Banjul).

Aunado a lo anterior, el artículo 15 del Pacto de Derechos Económicos, Sociales y Culturales ha de interpretarse en conjunto con instrumentos de soft law ${ }^{6}$, como son la Declaración de Friburgo sobre derechos culturales de 2007 y la Declaración de la Unesco de 2001 sobre diversidad cultural.

No obstante, en el último acuerdo multilateral en materia de propiedad intelectual -el Acuerdo sobre los Aspectos de los Derechos de Propiedad Intelectual Relacionados con el Comercio (ADPIC) de 1994- el cual es obligatorio para todos los miembros de la OMC, se excluyó de protección a los derechos morales de autor al no aplicar en el artículo 9 el artículo 6 Bis del Convenio de Berna. Finalmente, como lo menciona el profesor Diego Guzmán, en la legislación de los Estados Unidos se permite renunciar a estos derechos de conformidad con lo establecido en "la 106A del Copyright Act" (2018, p. 12).

\section{Atributos de los derechos morales de autor}

El derecho de paternidad y el derecho de integridad, reconocidos a nivel internacional, tienen características únicas que los diferencian entre sí:

\section{Derecho a la paternidad}

Este derecho se encuentra fundamentado en la teoría de la personalidad de Hegel y Kant. La obra se erige como una manifestación de la personalidad del autor: empreinte de la personnalité (huella de la personalidad); lo que significa que el autor tiene el derecho a reivindicar la paternidad de su obra y darse a conocer como el padre de ella. En virtud de este derecho subjetivo, el autor puede exigir que su nombre sea mencionado en cualquier soporte de su obra, y puede elegir ser reconocido por un seudónimo o permanecer en el anonimato (Raizon, 2014), sin importar el tipo de obra o el mérito artístico.

La protección al derecho de paternidad es tan amplia que, en diversas legislaciones, se exige ser respetado aun cuando se utilicen las excepciones o limitaciones al derecho de autor. Así, por ejemplo, el artículo 122-5 del Código de la Propiedad Intelectual de Francia exige mencionar el nombre del autor y la fuente en uso de las excepciones. Asimismo, el artículo 31 de la Ley 23 de 1982 de Colombia señala que: "en cada cita deberá mencionarse el nombre del autor de la obra citada y el título de dicha obra" y en el mismo sentido lo establece la Decisión Andina 351 de 1993 en el artículo 22.

Como resultado de la regulación, este derecho ha sido accionado en diversas jurisdicciones. Por ejemplo, la Cámara Nacional de Apelaciones en lo Civil de Argentina, en la sentencia del 4 de julio de 2011, condenó a Kapelusz Editora S.A a favor del Señor Jorge Wozniak por haber omitido mencionarlo como coautor en la portada de los Manuales de Ciencias Sociales de los grados 4, 5, 6 y, en consecuencia, condenó a la editorial a pagar daños y perjuicios por vulneración al derecho moral de paternidad. Igualmente, esta misma jurisdicción, en la sentencia del 18 de noviembre de 2010 condenó a Arte Gráfico Editorial Argentino S.A por haber publicado una fotografía inédita sin autorización del autor y además atribuida a un tercero en el diario el Clarín de fecha 14 de abril del año 2007. El fallo protegió el derecho moral a la paternidad e ineditud de la obra; en la sentencia se indicó que:

La omisión del nombre y más aún, la atribución de la obra a un tercero importa un agravio a su derecho de ser vinculado a la obra. Tanto para los autores, cuanto para los artistas o intérpretes en general la publicación de su nombre con sus características peculiares; lugar, tamaño de las letras, etcétera, presenta gran interés, toda vez 
que de ello dependerá en gran medida, no solo la posibilidad de lograr nuevas contrataciones y la explotación económica de su repertorio autoral, sino también de su desarrollo profesional (Conf. Lipszyc, Delia y Villalba, Carlos, "Derecho de los autores de obras fotográficas. Dando y reparación", ED, 121-645 citado en CNCiv., Sala J, "Villalobos, Horacio Pedro c. Arte Gráfico Editorial Argentino S.A. y otro", 22/11/2005, publicado en La Online), máxime cuando como en el caso, se encuentra ampliamente acreditado que el actor hace de la fotografía su profesión (ver contestaciones de oficio de fs. 186,198 y 217 y testimonios prestados a fs. 181/182, 183 y 184/185) 7 .

\section{Derecho a la integridad}

En virtud de este derecho, el autor tiene la facultad de impedir que su obra sea deformada, mutilada u objeto de cualquier otra modificación que pueda causar perjuicio a su honor o reputación. Así lo consagra el artículo 20 de la Ley italiana Protezione del diritto d'autore e di altri diritti connessi al suo esercizio. En Colombia, este derecho está reconocido en el artículo 30, literal b, de la Ley 23 de 1982. En cuanto a su regulación por la Comunidad Andina, este derecho se reconoce en el artículo 11, literal c, de la Decisión 351 de 1993.

Si bien la ley protege la obra ante cualquier alteración, ciertas modificaciones son legítimas, verbigracia las correcciones de estilo y de ortografía que efectúan las editoriales.

No obstante, si tales correcciones llegasen a dañar la integridad de una creación (situación que a veces ocurre con facilidad en la corrección de géneros como el de la poesía), evidentemente se podría incurrir en una violación a este derecho, por lo que siempre es recomendable consultar al autor sobre los cambios formales que se realicen a la obra. (Castro, 2006, p. 161).

La vulneración a este derecho moral de autor puede conllevar una condena en indemnización de perjuicios. (Villaba, 2019).

Un caso interesante respecto de la protección del derecho de integridad se presentó en la Corte de Casación Sala Civil de Francia del 12 de junio de 2001: los herederos de Antoine de Saint-Exupéry demandaron la adaptación al cine del libro El Principito, aun cuando habían celebrado un contrato de adaptación, por considerar que la película era una desnaturalización que vulneraba el derecho moral a la integridad de la obra. $\mathrm{Al}$ respecto, el fallo reconoció la libertad que le asiste al que hace la adaptación de la obra siempre y cuando se reproduzca fielmente la trama del libro. En este sentido, la respuesta de la Corte indicó que la celebración de un contrato de adaptación no implica de ninguna manera la renuncia al derecho al respeto de la obra; no obstante, la adaptación al cine de una obra literaria implica que se reconozca cierta libertad al adaptador. Adicionalmente, la Corte indicó que soberanamente la obra adaptada respetó el espíritu de la obra preexistente y que, aunque incluía una contribución personal del adaptador - requerida por la transposición en la pantalla-, esta reproducía fielmente la trama y el carácter del personaje principal.

\section{La Protección del derecho moral en Colombia y la legislación andina}

El ordenamiento jurídico colombiano en materia autoral se caracteriza por proteger una dualidad de derechos. Respecto al derecho moral, el ordenamiento jurídico colombiano otorgó mayor protección que aquella establecida por el Convenio de Berna. De esta manera, la Ley 23 de 1982 reconoce la existencia de cinco derechos morales: paternidad, integridad, modificación, retracto e ineditud.

El derecho de ineditud, consagrado en el artículo 30, literal c, de la Ley 23 de 1982 y en el artículo 11, literal a, de la Decisión 351 de 1993, hace referencia al derecho del autor de decidir si su obra es publicada o no. De esta manera, el autor es el que decide en qué momento juzga oportuno y en cuáles circunstancias publicará su obra (Raizon, 2014). Ahora bien, es importante aclarar que la ineditud no se afecta por la comunicación privada de la obra ni afecta a un grupo de personas cercano; es necesario que la obra se haga accesible al público ya sea al ser divulgada o publicada (Anaya Quintero y Cruz Fino, 2018).

El derecho de arrepentimiento o retracto, por su parte, se refiere al derecho que le permite al autor retirar la obra del mercado, incluso si esta ha sido publicada. En virtud del artículo 30 de la Ley 23 de 1982, literal e y párrafo 4, para ejercer este derecho, se exige que previamente se indemnice a los terceros por los perjuicios que se les haya podido ocasionar.

La duración de la protección establecida para estos derechos en la Comunidad y en el país es mucho mayor que la ordenada por Berna. La Decisión Andina 351, por ejemplo, establece de manera clara que los derechos morales permanecen en 
el tiempo con el autor y, determina que estos son inalienables, inembargables, imprescriptibles e irrenunciables. Por su parte, el artículo 30 de la Ley 23 de 1982 de la legislación colombiana establece que dichos derechos son perpetuos. Dentro de esta legislación, la protección a los derechos morales de autor solo se encuentra sujeta a dos excepciones y limitaciones. En primer lugar, en obras arquitectónicas, donde el autor no podrá impedir que el propietario introduzca modificaciones a esta (Ley 23 de 1982, art. 43). En segundo lugar, en las obras creadas por funcionarios públicos; estas personas solo podrán ejercer sus derechos morales cuando no sea incompatible con los derechos y obligaciones de la entidad pública afectada (Ley 23 de 1982, art. 91).

Adicionalmente, el ordenamiento jurídico colombiano ha reconocido a los derechos morales la categoría de derechos fundamentales. Así lo determinó la Corte Constitucional en Sentencia C-155 de 1998 al considerar que, dado que la función creadora del ser humano es inherente a su condición de ser racional, desconocer la condición de autor equivale a desconocerle al hombre su condición de ser pensante. Con esta declaración, la Corte está protegiendo los derechos morales más allá de lo requerido por el Convenio Berna (Bernal y Conde, 2017). En la práctica, esta clasificación tiene efectos importantes: primero, estos derechos pueden ser protegidos por la acción de tutela en virtud de lo consagrado en el artículo 86 de la Constitución Política. Segundo, cualquier tratado internacional que verse sobre derechos morales de autor se integra al bloque de constitucionalidad. Por ejemplo, la Corte Constitucional en sentencia c-582 de 1999 estableció que tanto la Decisión Andina como el Convenio de Berna conformaban el bloque de constitucionalidad, no siendo el mismo caso para el ADPIC. Como tercero efecto, durante la interpretación de estos derechos, se pueden utilizar principios propios de la protección de los derechos humanos como el principio pro homine (Corte Suprema de Justicia, 2010).

Finalmente, existen en el ordenamiento colombiano acciones penales y civiles disponibles para el autor para proteger cualquier infracción al derecho moral. Estas acciones se han utilizado efectivamente. Por ejemplo, con la Sentencia 31403 de 2010, la Corte Suprema de Colombia condenó a una profesora a dos años de cárcel por haber utilizado algunos extractos de la tesis de una estudiante en su propia obra sin la debida citación (Corte Suprema de Justicia, 2010).
Este caso revela cómo el derecho autoral doméstico y regional en materia de derecho de autor se caracteriza por otorgar una protección que supera los mínimos estándares establecidos internacionalmente y que, incluso, comparadamente, otorga más derechos que otros ordenamientos en los cuales los derechos morales se caracterizan por ser inherentes al ser humano.

\section{El ordenamiento jurídico andino y las interpretaciones prejudiciales}

La Comunidad Andina (CAN), como una integración y cooperación entre países en busca del desarrollo económico y social armonioso y equilibrado, tiene sus inicios en 1996 con la reforma introducida por el Protocolo de Trujillo al Acuerdo de Cartagena (Palacio, 2013). Sin embargo, anterior a dicha fecha, el Acuerdo de Cartagena de 1969 tenía un objeto más limitado: establecer un mercado común (Jiménez, 2013). Inicialmente, la CAN fue integrada por cinco países latinoamericanos: Colombia, Bolivia, Ecuador, Perú y Venezuela, pero en el 2005, este último decidió retirarse de Comunidad (BBC, 2006).

La CAN, con miras a lograr la integración regional, creó una serie de órganos e instituciones para promover el proceso, los cuales fueron enumerados en el artículo 6 del Acuerdo de Cartagena. Asimismo, la Comunidad estableció su propio ordenamiento jurídico conformado por dos tipos de normas: a) las primarias u originarias, tales como los Tratados constitutivos de la CAN y sus organismos, como el Acuerdo de Cartagena y, b) la normas derivadas o secundarias, que corresponden a aquellas normas dictadas por los órganos andinos según su competencia en forma de decisiones o resoluciones (Tremolada, 2006).

El ordenamiento jurídico comunitario se caracteriza por ser de aplicación directa y efecto inmediato en el sistema jurídico de los países miembros; por lo tanto, dichas normas deben ser aplicadas internamente por los jueces desde su promulgación (Proceso 57-IP-2012). La normativa también se caracteriza por su primacía; es decir que la norma andina prevalece sobre cualquier otra norma de derecho interno que pueda ser contraria o que de algún modo obstaculice su aplicación (Tratado de Creación del Tribunal de Justicia de la Comunidad Andina - TCTJC, artículo 4).

La interpretación de la norma andina ha sido encomendada al Tribunal Andino de Justicia, 
órgano jurisdiccional de la Comunidad (artículo 40, Acuerdo de Cartagena). Este órgano, entre otras funciones, tiene la labor de promover y asegurar la aplicación uniforme de las normas que conforman el ordenamiento jurídico de la Comunidad, a través de la interpretación prejudicial (TCTJC, artículo 32). En otras palabras, la interpretación prejudicial permite que las normas andinas tengan el mismo alcance en todos los países miembros.

Dos características de la interpretación prejudicial permiten alcanzar este propósito. Primero, se debe solicitar de manera obligatoria de oficio o a petición de parte en aquellos casos donde deba aplicarse o se controvierta alguna norma de la Comunidad, siempre que la sentencia no sea susceptible de recursos en el derecho interno. En este caso, el juez suspenderá el procedimiento hasta haber recibido la interpretación del Tribunal para poder dictar sentencia, (TCTJC, artículo 34). Segundo, el juez nacional que esté conociendo el asunto deberá adoptar en su sentencia la interpretación del Tribunal (TCTJC, artículo 35).

Respecto de la primera característica, se puede precisar que en un proceso de única instancia y el juez de segunda instancia -incluso en los casos que sean susceptibles del recurso extraordinario de casación-, los jueces se encuentran obligados a solicitar la interpretación prejudicial de la norma andina antes de fallar (Perotti, 2016). En caso en que el juez nacional se abstenga de solicitar la interpretación prejudicial, o habiéndola solicitado aplique una interpretación diferente a la otorgada por el Tribunal, los países miembros o los particulares podrán hacer uso de la acción de incumplimiento (Decisión 500 de 2001, artículo 128). Esta norma busca que un país miembro dé cumplimiento a las obligaciones y compromisos contraídos como miembro de la Comunidad Andina.

Es decir, el alcance y contenido que el TJCA otorgue al ordenamiento jurídico a través de su interpretación deberá ser el mismo que utilice el juez nacional en su sentencia. Ahora, es menester aclarar que el TJCA solo se referirá a la normativa andina, no a la legislación doméstica, ni resolverá el caso como tal (artículo 34, TCTJC). Para la Corte Constitucional Colombiana, tener una interpretación unificada de la normativa andina va acorde con el principio de igualdad, ya que de este principio se deriva que la aplicación de las normas que componen este ordenamiento jurídico se realice de manera homogénea. (sentencia C-277, 1999). De lo contrario, existirían tantas interpretaciones como jueces existentes en los diversos países miembros. No obstante, en los casos donde la sentencia es susceptible de recursos, será facultativo para el juez solicitar la interpretación prejudicial. En este caso, el proceso no se suspende y el juez deberá dictar sentencia incluso si no ha llegado la interpretación del Tribunal (artículo 34, TCTJC).

Al respecto de cómo el TJCA podrá realizar la interpretación del derecho comunitario, el Acuerdo 8 de 2017 del Tribunal de Justicia de la Comunidad Andina -el cual reguló varios aspectos vinculados al ejercicio de la interpretación prejudicial- brindó ciertas luces respecto de los métodos de interpretación del derecho comunitario andino. Dicho acuerdo establece que se podrán utilizar los diferentes métodos de interpretación reconocidos por la Teoría General del Derecho -teniendo en cuenta la realidad y las características esenciales del derecho comunitario- y emplear, de preferencia, los métodos de interpretación funcionales tales como el sistemático, el teleológico y de la ratio legis (artículo 4). De esta manera, la interpretación otorgada por el TJCA es el llamado a determinar el verdadero alcance de la normativa comunitaria en los países miembros, la cual deberá ser aplicada por los jueces nacionales.

\section{La interpretación prejudicial y el derecho moral de integridad, caso 47-IP-17}

La interpretación prejudicial 47-IP-17 es un caso enigmático en cuanto a la interpretación de los derechos morales de autor por ser el primer escenario en que el TJCAN -alejándose de un simple criterio de interpretación gramatical- hace una ponderación de derechos entre el derecho moral de autor y el derecho de propiedad, en situaciones donde la creación intelectual se encuentra plasmada sobre un soporte físico de propiedad de un tercero. De igual manera, el Tribunal, al margen de la discusión, realiza una ponderación con otros derechos tales como el interés y seguridad pública, y otros derechos fundamentales, como el derecho a la imagen o intimidad de las personas.

\section{Antecedentes}

La interpretación fue solicitada por el Tribunal Superior del Distrito Judicial de Bogotá por ser el Tribunal de cierre en el caso Gabriel Antonio Calle vs. Centro Comercial San Diego. Este caso cons- 
tituyó el primero en ser resuelto por la Dirección Nacional de Derecho de Autor (DNDA) de Colombia bajo sus funciones jurisdiccionales otorgadas bajo la Ley 1564 de 2012. El Tribunal Superior de Bogotá solicitó a el TJCA, entre otros, la interpretación del literal c del artículo 11 de la Decisión Andina 351 referente al derecho moral de integridad.

En el caso Gabriel Antonio Calle vs. Centro Comercial San Diego se presentaron los siguientes hechos: Gabriel Calle fue un artista contratado en el 2006 por el Centro Comercial San Diego, ubicado en la ciudad de Medellín, para elaborar un mural representativo de las costumbres y valores de la región antioqueña en la fachada occidental del Centro Comercial. Bajo esos presupuestos el demandante elaboró el mural titulado "Líder". Siete años después, en el 2013, el mural presentaba deterioros debido al paso del tiempo y al estar ubicado en la intemperie. Ante ello, el artista ofreció al Centro Comercial realizar la restauración sin costo alguno. No obstante, el Centro Comercial no accedió a dichas peticiones y en la segunda mitad del mismo año borró el mural.

Con base en estos sucesos, el demandante presentó un proceso verbal ante la DNDA durante el cual argumentó que el Centro Comercial había infringido sus derechos morales de autor y solicitó una indemnización de 170 millones de pesos y que se ordenara realizar de nuevo la obra artística "Líder". Por su parte, el Centro Comercial argumentó que la supresión del mural obedeció a un caso de necesidad, ya que no solo el mural se encontraba deteriorado, sino que también el soporte físico amenazaba ruina; que había actuado de buena fe al consultar a profesionales y a la Secretaría de Cultura Ciudadana de Medellín y que, finalmente, como cualquier obra a la intemperie estaba destinada a desaparecer.

La DNDA, en agosto de 2016, expidió la decisión de primera instancia en la cual efectivamente declaró infringido el derecho de integridad del demandante por parte del Centro Comercial. La Dirección consideró que borrar la obra "Líder" representaba una deformación de ella, y se había atentado contra el decoro de la misma al haber eliminado "la pureza, el recato y la estimación de la manifestación artística que realizó el autor, de una forma tal que será imposible volver a percibir la misma pese a que esta pueda ser representada a través de otros soportes como fotográficos" (Dirección Nacional de Derecho de Autor, 2016, p. 8).
Respecto a la tensión frente al derecho de integridad y derecho de dominio, la DNDA estableció que se debía resolver el caso a través de un respeto mutuo, lo que se traduce en que el titular del derecho de propiedad debe procurar no afectar el derecho de integridad, y el autor no puede utilizar un soporte sin autorización del titular del dominio, limitando el derecho de dominio a través de la realización de una obra sin autorización. En el caso en concreto, el juez encontró que el demandante había realizado la obra con autorización del titular del derecho de dominio y, por lo tanto, este último estaba destinado a respetar el derecho de integridad (Dirección Nacional de Derecho de Autor, 2016).

Finalmente, la DNDA condenó al pago de cien (100) Salarios Mínimos Legales Mensuales Vigentes, pero no accedió a la pretensión de realizar de nuevo la obra. A la presente decisión, el Centro Comercial presentó un recurso de apelación. El Tribunal Superior de Distrito Judicial, tribunal de segunda instancia en casos relativos al derecho de autor, solicitó la interpretación prejudicial al TJCA.

\section{Consideraciones del TJCA}

El TJCAN, una vez conocida la solicitud de interpretación, empezó por establecer que, efectivamente, los derechos morales consagrados en el artículo 11 de la Decisión son inalienables, inembargables, imprescriptibles e irrenunciables. El texto de esta norma implica que dichos derechos son ilimitados en el tiempo. No obstante, su interpretación no se limita a aplicar un criterio de interpretación gramatical.

El TJCA aclaró que, con base en el derecho moral de integridad (derecho en que se centra la interpretación), una obra no puede ser modificada, mutilada o deformada sin autorización del autor. No obstante, el Tribunal también indicó que dicho derecho no es absoluto y, por lo tanto, cuando se encuentre en conflicto con otros derechos, se debe realizar una ponderación. En este sentido, se señala que un escenario donde se puede evidenciar el conflicto es el caso en que la obra se encuentre plasmada en un soporte material de propiedad de un tercero cuando sea muy difícil o imposible separar la obra del soporte material y, por lo tanto, la suerte del soporte material será la misma que la de la obra (47-IP-17, párr. 3.2).

De esta manera, el TJCA empieza a establecer criterios para guiar en un caso concreto cómo se deberá resolver el conflicto entre el derecho de 
propiedad y el derecho moral de autor. El primer paso, según el Tribunal, es considerar si existió un acuerdo previo entre las partes. De ser afirmativo, las afectaciones o modificaciones al soporte material que afecten la obra se regirán por lo pactado entre las partes. Dicho acuerdo seguirá aplicando para ventas posteriores del soporte material.

$\mathrm{Si}$ se trata de un conflicto sobre aspectos que no fueron pactados contractualmente, se debe buscar un equilibrio entre el derecho moral de integridad y el derecho de propiedad sobre el soporte material, considerando unos criterios enunciativos que el Tribunal establece:

a) Obra realizada sin autorización o en extralimitación de las indicaciones. Si se trata de una obra elaborada sin autorización del propietario del soporte material, este -a su discreción- podrá decidir conservarla o eliminarla e incluso modificarla, mutilarla o deformarla, sin que haya posible infracción al derecho de integridad. Lo mismo sucedería, si a pesar de ser una obra por encargo, el autor extralimitó las indicaciones expresas de quien encargó la obra (47-IP-17, párr. 4.2.1).

b) El material de la obra no duradero. En caso de obras plasmadas realizadas con un material no duradero en un soporte material de un tercero, el autor tiene conocimiento de que la misma está destinada a sufrir daños o a desaparecer con el tiempo. Este es el caso de una escultura de hielo o de arena. (47-IP-17, párr. 4.2.2).

c) Carácter permanente o temporal de la obra. Se debe determinar si es una obra elaborada con la intención de permanencia y que no sea afectada por factores externos, o si es una obra elaborada con la consciencia -tanto por parte del autor como de terceros- de que será temporal ya que por su propia naturaleza está destinada a sufrir deterioro, destrucción o consumo en un corto plazo. Se puede tratar de obras creadas para acontecimientos específicos como festivales, o con fines específicos decorativos según el gusto del propietario, o aquellas con fines comerciales, cuya permanencia viene vinculada a la finalidad por la cual fue creada. (47-IP-17, párr. 4.2.3).

d) Interés público y seguridad pública. Si el derecho de integridad del autor entra en conflicto con el interés público, debería primar el interés público por ser de carácter general sobre un interés particular. De igual manera, el Tribunal establece que si la obra se encuentra en un soporte material que pueda afectar la seguridad pública, debe primar la seguridad pública siempre que esté acreditado el riesgo a la seguridad (47-IP-17, párr. 4.2.4).

e) Riesgo de deterioro o pérdida de la propiedad. En aquellos casos donde el soporte material, como un muro o estructura, requiera una intervención para su conservación, el propietario podrá realizar las modificaciones necesarias al soporte. No obstante, con anterioridad deberá informar al autor para efectos de permitirle el retiro de la obra, de ser posible, o tomas de muestras fotográficas u otras muestras que el autor considere pertinente. Lo anterior no será aplicable en caso fortuito o fuerza mayor (47-IP-17, párr. 4.2.5).

f) Afectación a derechos de terceros. En aquellos casos donde se deba modificar una obra en aras de cautelar los derechos fundamentales de un tercero, como el derecho a la imagen y buen nombre, cuando haya sido incluido, por ejemplo, su imagen sin autorización, se podrán hacer las modificaciones sin afectar el derecho de integridad (47-IP-17, párr. 4.2.6).

g) Medidas diligentes del propietario para la conservación de la obra: Dado que, ante el conflicto entre el derecho de propiedad y el derecho de integridad, se debe cautelar en la medida de lo posible el derecho de integridad, el propietario del inmueble deberá tomar las acciones necesarias para informarle al autor la futura afectación de manera que este último pueda tomar medidas como retirar el soporte material de ser posible, tomar fotografías, filmar o efectuar cualquier otra acción que ayude a conservar la obra. Esto no será necesario en el caso de seguridad, caso fortuito o fuerza mayor (47-IP-17, párr. 4.2.6).

De esta manera, mediante el establecimiento de una serie de criterios de ponderación enunciativos en casos donde exista conflicto entre el derecho de integridad y el derecho de propiedad sobre el soporte material, el TJCA resolvió la consulta realizada por el Tribunal Superior de Bogotá. Asimismo, el TJCA determinó ciertos criterios de ponderación con otros derechos.

\section{Aplicación de la interpretación 47-IP-17 por el Tribunal Superior de Bogotá}

El 2 de noviembre de 2017, el Tribunal Superior de Bogotá dictó la sentencia de segunda instancia en el caso de Gabriel Antonio Calle vs. Centro Comercial San Diego, dando cumplimiento a la interpretación 
47-IP-17. La sentencia revocó el fallo de primera instancia de manera tal que no solo se denegaron las pretensiones, sino que se condenó al demandante en costos.

Al abordar el problema jurídico, el Tribunal Superior de Bogotá utilizó dos de los criterios establecidos por el TJCA para ponderar el derecho de propiedad sobre el soporte material y el derecho de integridad para resolver la tensión: 1) El carácter permanente o temporal de la obra y 2) El riesgo de deterioro o pérdida de la propiedad.

Respecto del primero, el Tribunal -luego de analizar los hechos entorno a la realización de la obra y la naturaleza del soporte material- consideró que la obra no estaba destinada a la permanencia. Al ser una obra que fue pintada a la intemperie y en un Centro Comercial que, por cuya naturaleza, está destinado a sufrir cambios en el tiempo, y dado que la obra fue específicamente encargada con un objetivo de decoración para un evento comercial específico, el Tribunal concluyó que tanto el autor como terceros sabían que la obra iba a ser temporal. Respecto al segundo criterio, el Tribunal consideró que se encontraba probado en el proceso que el soporte material se encontraba deteriorado, que había un riesgo de que colapsara y que significaba una amenaza para la seguridad pública (Tribunal Superior de Bogotá, min. 30 al 39).

De esta manera, al aplicar los dos criterios, ambos pesaron a favor del titular del derecho de propiedad sobre el derecho de integridad, lo que llevó al Tribunal a revocar la decisión de primera instancia. Sin embargo, el Tribunal no explica por qué solo elige esos dos criterios.

\section{Resultado: la flexibilización del derecho moral en el ámbito doméstico a través de la jurisprudencia andina}

El derecho moral de autor, como se describió anteriormente, por proteger la conexión espiritual entre autor y obra es de carácter personalísimo y se encuentra fuera del comercio al no poderse disponer de él, situación que expresa la literalidad de la ley colombiana y andina al decir que el derecho moral es inalienable, inembargable, imprescriptible e irrenunciable" No obstante, se debe observar cómo la decisión 47-IP-17 el TJCAN se aparta de la literalidad de la misma norma andina y, mediante de un criterio hermenéutico conflictivista ${ }^{8}$ - utilizado principalmente en la interpretación de textos constitucionales-, sugirió que en efecto hay ciertos escenarios en los cuales el derecho moral de integridad puede ser, a lo menos, renunciable. Estas decisiones devienen del primer paso establecido para resolver un conflicto entre el derecho de dominio y de integridad, esto es: la existencia de un acuerdo que regule el tema entre las partes, sugiriendo que existe una posibilidad válida de que el autor disponga de su derecho de integridad.

Adicionalmente, los criterios de ponderación enunciados por el TJCA crean, de cierta forma, limitaciones temporales a un derecho que se reputaba perpetuo por la misma legislación colombiana (Ley 23 de 1982 art. 30). Un ejemplo es al establecer como criterio de ponderación entre ambos derechos, el propósito específico para el cual se ha creado la obra, indicando que hay obras que no tienen vocación de perdurar. No obstante, el hecho de que una obra se piense para un evento específico no siempre impacta en la posibilidad que tiene la obra de perdurar en el tiempo, especialmente cuando el mismo artista la puede restaurar; por lo tanto, la decisión establece un límite artificial al derecho de integridad.

De esta forma, se puede ver cómo la posición del Tribunal Andino sobre la protección del derecho moral de integridad es mucho más flexible que la establecida en el derecho doméstico. El ejemplo más claro de esta situación es el caso de Gabriel Antonio Calle vs. Centro Comercial San Diego, donde, en primera instancia -basados en una interpretación literal de la norma doméstica y andina-, el demandado fue condenado por infringir el derecho de integridad al haber atentado contra el decoro de la obra; lo que llevó al juez a determinar que el propietario tenía el deber de respetar el derecho de integridad. No obstante, en segunda instancia, basado en la interpretación andina, el Tribunal vio la necesidad de denegar las pretensiones al considerar, entre otras, que la obra se reputaba temporal.

Dado que los jueces domésticos deben acoger las interpretaciones prejudiciales, so pena de la acción de incumplimiento, son dichas decisiones las que determinan el ámbito de protección del derecho de autor. De esta manera, luego de la decisión 47-IP17, el derecho moral de integridad para obras que por su naturaleza existan en un soporte material de titularidad de un tercero y cuya separación no sea posible sin afectar alguno de los dos -como lo puede ser una obra arquitectónica, un mural en pared ajena, una escultura en material ajeno entre otras-, 
debe pensarse como un derecho renunciable. Como tal, el derecho podría ser temporal y cuya protección debe ceder en casos donde el interés público, la seguridad, el derecho a la imagen de un tercero y la pérdida de la cosa estén en juego. De igual manera, es un derecho que no se puede accionar en caso de que la obra sea ilegal; es decir, cuando no se haya obtenido autorización para utilizar el soporte.

Aunque es cierto que las interpretaciones prejudiciales solo rigen para el caso en concreto y, por lo tanto, la existencia de una decisión frente a un caso similar no exime al juez nacional de solicitar dicha interpretación, el papel de dichas interpretaciones es buscar una aplicación armoniosa de la normativa. Por consiguiente, el propósito es formar una línea jurisprudencial uniforme, tal como lo reconoció el TJCA en el proceso 1-IP-87. Esta situación se ve reflejada en la jurisprudencia andina cuando decisiones pasadas son frecuentemente citadas en nuevos casos para sustentar la nueva decisión. No obstante, eso no indica que el Tribunal no pueda cambiar de posición cuando encuentre razones justificadas para hacerlo (proceso 7-IP-89).

De esta manera, el entendimiento que se deberá otorgar al derecho moral de integridad será el establecido por la TJCA hasta que encuentre razones para que dicha posición sea modificada. De lo contrario, querer dar aplicación a un derecho de integridad absoluto podrá conllevar a la revocatoria de la decisión o dará lugar a una acción de cumplimiento.

\section{Conclusión}

El derecho moral de autor por proteger la conexión espiritual entre autor y obra es de carácter personalísimo y se encuentra fuera del comercio al no poderse disponer de él, situación que expresa la literalidad de la ley colombiana y andina al decir que el derecho moral es inalienable, inembargable, imprescriptible e irrenunciable. No obstante, a pesar de la literalidad de la norma andina, el verdadero significado de esta se encuentra a cargo del Tribunal de Justicia Andino, quienes son el único ente encargado de la interpretación de la norma andina en aras de una aplicación armoniosa de esta en los países miembros. Tal como lo establece la Decisión 500 de 2017, el Tribunal Andino de Justicia propende por criterios hermenéuticos funcionales, tales como el sistemático, el teleológico y de la ratio legis, al momento de resolver las interpretaciones de los Estados miembros.
El caso 47-IP-17 es un claro ejemplo de la hermenéutica utilizada por el Tribunal. En dicho caso, el TJCAN estableció unos criterios de ponderación entre el derecho de dominio y el derecho de integridad, lo que lleva a sugerir que se flexibilizó el derecho moral de integridad para obras que por su naturaleza existan en un soporte material de titularidad de un tercero y cuya separación no sea posible sin afectar alguno de los dos, como lo puede ser una obra arquitectónica, un mural en pared ajena, una escultura en material ajeno entre otras. Ante estas circunstancias, este derecho debe pensarse como un derecho renunciable que podría ser temporal y cuya protección debe ceder en casos donde el interés público, la seguridad y el derecho a la imagen de un tercero, y la pérdida de la cosa estén en juego. De igual manera, es un derecho que no se puede accionar en caso de que la obra sea ilegal; es decir, cuando no se haya obtenido autorización para utilizar el soporte.

Adicionalmente, dado que las interpretaciones prejudiciales son de obligatorio cumplimiento por el juez doméstico, las decisiones tomadas por el TJCA impactan el derecho nacional, tal como se puede evidenciar al comparar la decisión de primera y segunda instancia en el caso Gabriel Antonio Calle vs Centro Comercial San Diego.

\section{Referencias}

Gobiernos de Bolivia, Colombia, Ecuador y Perú. (1996). Acuerdo de Integración Subregional Andino (Acuerdo de Cartagena). Trujillo, Perú. https:// idatd.cepal.org/Normativas/CAN/Espanol/Acuerdo_de_Cartagena.pdf

Almansi, C., Baggi, M., Contel, R., Cottier, B., y Werra, J. (2010). Manuale Il diritto d’autore nellinsegnamento. Lugano \& Genève. http://www.diceproject. ch/wp-content/uploads/2011/05/Handbook_web_ it.pdf

Anaya Quintero, L. A., y Cruz Fino, J. A. (2018). Arbitrabilidad de los derechos morales de autor: estudio desde la perspectiva del arbitraje comercial internacional. Universidad Externado de Colombia.

Bernal, D. y Conde, C (2017). Los derechos morales de autor como derechos fundamentales en Colombia. Revista la Propiedad Inmaterial, 24(2), 53-66 https://doi.org/10.18601/16571959.n24.03

Bernal, E. (2016). La libertad de expresión en la internet. Misión Jurídica, 9(10), 163-180.

Bernal, E. (2019). Internet y la paradoja de la libertad. Blog Departamento de Propiedad Intelectual, Universidad Externado de Colombia. https://propintel. 
uexternado.edu.co/internet-y-la-paradoja-de-la-libertad/

Berthier, P. (1936). La Protection légale du compositeur de musique. Rousseau.

Binctin, N. (2013). Le droit moral en France. Les Cahiers de propriété intellectuelle, 25(1). https://www.lescpi.ca/s/666.

Busaniche, B. (2019). Clase 1: La propiedad intelectual en la Declaración Universal de los Derechos $\mathrm{Hu}$ manos. Maestría en Propiedad Intelectual, Flacso Argentina.

Busaniche, B. (2019). Clase 2: Interpretación y aplicación del Pacto de Derechos Económicos, Sociales y Culturales. Maestría en Propiedad Intelectual Flacso Argentina.

Corte Suprema de Justicia. (2010). Sentencia de Casación No 31403 de 28 de mayo de 2010. M.P: Sigifredo Espinosa Pérez

Desbois, H. (1960). Les Conventions de Berne (1886) et de Genève (1952) relatives à la protection des œuvres littéraires et artistiques. Annuaire français de droit international, 6, 41-62. https://www.persee. fr/doc/afdi_oo66-3085_196o_num_6_1_895

Consejo Andino de Ministros de Relaciones Exteriores. (2001). Decisión 500 de 2001, Estatuto del Tribunal de Justicia de la Comunidad Andina.

Dirección Nacional de Derecho de Autor. (2016). Gabriel Antonio Calle Arango vs. Centro Comercial Sandiego P.H. Asuntos Jurisdiccionales.

Guzmán, D. (2018). Derecho del arte: El derecho de autor en el arte contemporáneo y el mercado del arte. Universidad Externado de Colombia.

Hegel, G. W. F. (2012). Principios de la filosofía del derecho. Sudamericana

Jiménez, W. G. (2013). Papel de la jurisprudencia del tribunal de justicia de la comunidad andina en decisiones Judiciales de los Países Miembros, International Law: Revista Colombiana de Derecho Internacional, (23), 87-117.

Le Chapelier, I. R. G. (1791). Rapport fait par M. Le Chapelier, au nom du comité de constitution, sur la pétition des auteurs dramatiques, dans la séance du jeudi 13 janvier 1791, avec le Décret rendu dans cette séance. Imprimerie Nationale.

Naciones Unidas. (s.f.). Histoire de la rédaction de la DUDH. Disponible en https://www.un.org/fr/sections/universal-declaration/history-document/ index.html.

Palacio, P. M. (2016). Derechos de autor, tecnología y educación para el siglo XXI: El tratado de libre comercio entre Colombia y Estados Unidos. Universidad Sergio Arboleda.
Palacio, M. (2013). Conflicto de leyes al interior del régimen común de derechos de autor y derechos conexos de la Comunidad Andina de Naciones. Revista La Propiedad Inmaterial, 17, 205-222.

Raizon, H. (2014). La contractualisation du droit moral de l'auteur. [Tesis de doctorado, Université d'Avignon]. https://tel.archives-ouvertes.fr/tel-01205616

Shaver, L. (2010). The right to science and culture. Wisconsin Law Review, 121-184.

Tremolada, E. (2006). El Derecho Andino: una Sistematización Jurídica para la Supervivencia de la Comunidad Andina de Naciones, Cuadernos Constitucionales de la Cátedra Fadrique Frió Ceriol, (57), 35-75.

Parra, T. E. (2014). Introducción al Derecho Intelectual. Editorial Porrúa.

UNESCO. (2016). Comite̋ intergubernamental sobre la protección y la promoción de la diversidad de las expresiones culturales.

Perotti, A (2016) Algunas consideraciones sobre la interpretación prejudicial obligatoria en el derecho andino. Díkaion, 16(11), 128-153.

Vega, J. A. (2010). Manual de derecho de autor. Bogotá: Dirección Nacional de Derecho de Autor Unidad Administrativa Especial Ministerio del Interior y de Justicia.

BBC (2006, abril 20). Venezuela sale de la CAN. BBC Mundo http://news.bbc.co.uk/hi/spanish/latin america/newsid_492400o/4924980.stm.

Villalba, F. (2019). Clase 3. El contenido del derecho de autor. Los derechos morales y patrimoniales. Maestría en Propiedad Intelectual Flacso Argentina.

Zucconelli, G. (2014). Il diritto d'autore nella prospettiva europea. [Dottorato di Ricerca in Diritto dell'Unione Europea. Università degli studi di Ferrara]. https://core.ac.uk/download/pdf/33162439.pdf

\section{Notas}

Ver, por ejemplo: Dirección Nacional de Derecho de Autor (DNDA) (2018), Concepto: Competencia. Generalidades - Objeto de Protección - Alcance de las facultades exclusivas - Derecho de Transformación - Obras derivadas - Licencias. Radicado: 1-2018-22180. Disponible en http://200.91.225.128/ Intrane1/desarrollo/CONCEPTOSWEB/arch_conceptos/1-2018-22180.pdf

2 Sobre este punto existe un importante pronunciamiento de la Honorable Corte Constitucional mediante la sentencia C-234/19, en la cual se estudió la exequibilidad del artículo 538 numeral 3 (parcial) del Decreto 410 de 1971, en un tema relacionado con 
patentes de invención. En este fallo, la Corte resolvió "declarar EXEQUIBLE el enunciado 'o a las buenas costumbres' del artículo 538, numeral 3, del Código de Comercio, bajo el entendido de que se remite al criterio de 'moral social' o 'moral pública'"

3 Sobre este punto, se aclara que la DNDA conoce en primera instancia los procesos por infracción de los derechos de autor de acuerdo con lo establecido en el Código General del Proceso; por lo tanto, es facultativa la solicitud de interpretación prejudicial. No obstante, luego de una revisión exhaustiva de las sentencias proferidas por esta entidad del Estado, hasta el momento no ha solicitado ninguna interpretación al Tribunal Andino de Justicia.

4 Así mismo, el derecho moral de autor se refiere a elementos inmateriales como la libertad, sensibilidad, imaginación y la belleza (Berthier, 1936, p. 148).

5 Ver l'exposé des motifs de la proposition de loi «tendant à compléter la loi des 19/24 juillet 1793» et la loi du14 juillet 1866 sur la propriété littéraire et artistique pour assurer la protection du droit moral de l'auteur rédigé par Plaisant, en DA 15, mayo 1921, p. 59, como se cita en Raizon, 2014, p. 21
${ }^{6}$ Respecto a los instrumentos mencionados de soft law, estos son pertinentes en materia de derecho de autor teniendo en cuenta la relación que se establece entre estos y el acceso a la cultura establecido en el artículo 5 de la Declaración de Friburgo, en el cual se menciona la protección de los derechos morales de autor. Respecto a la Declaración de la Unesco sobre diversidad cultural, el artículo 8 menciona el reconocimiento a los derechos de autor.

7 Disponible en http://aplicaciones.cerlalc.org/derechoenlinea/dar/index.php

8 Haciendo referencia a un criterio propio de interpretación de la ley que busca ponderar los intereses inmersos en ella y que es propio de la interpretación constitucional. Para más información sobre la interpretación conflictivista, ver Diego Medina, Interpretación Constitucional, Bogotá: Escuela Judicial "Rodrigo Lara Bonilla", (2006), p. 55 y ss. 